\title{
Cuidados paliativos para adolescentes com câncer: uma revisão da literatura
}

\author{
Palliative care to adolescents with cancer: a literature review \\ Cuidados paliativos para adolescentes con cáncer: una revisión de la literatura
}

\section{Patrícia Pereira Remedi', Débora Faleiros de Mello', Maria José Menossi', Regina Aparecida Garcia de Lima'}

'Universidade de São Paulo. Escola de Enfermagem de Ribeirão Preto. Ribeirão Preto, SP

Submissão: 16/07/2007

Aprovação: 20/12/2008

\section{RESUMO}

Cuidar de adolescentes com câncer no processo de morte e morrer é um desafio para os profissionais de saúde, pelo alto custo emocional e especificidades Que envolvem esta etapa do desenvolvimento humano. O objetivo deste estudo é revisar a produção científica relativa aos cuidados paliativos para adolescentes com câncer. Trata-se de uma pesquisa bibliográfica com coleta de dados nas bases Lilacs, Medline, Psyclnfo e vias não-sistemáticas. Identificaram-se três temáticas: adolescência e suas diferentes definições; particularidades do adolescente com câncer e cuidados paliativos para adolescente com câncer. Observou-se ausência de pesQuisas baseadas em evidências Que definam o panorama dos sintomas Que afetam a Qualidade de vida durante os cuidados paliativos, bem como ausência de programas específicos para esta fase de transformações rápidas Que, por si só, demanda esforços adaptativos.

Descritores: Adolescente; Neoplasia; Cuidados paliativos; Enfermagem pediátrica.

\section{ABSTRACT}

Providing care to adolescents with cancer in the process of death and dying has been a great challenge for health professionals. This challenge is marked by a high emotional burden and specificities of this stage of human development. The purpose of the present study was to review the scientific literature regarding palliative care to adolescents with cancer. This study is a literature review, which data collection was performed using Lilacs, Medline, and Psyclnfo, in addition to non-systematic databases. An analysis of the manuscripts revealed three themes: adolescence and its different definitions; the particularities of adolescents with cancer; and palliative care to adolescents with cancer. The study showed there is a scarcity of evidenced-based research defining the panorama of symptoms affecting the Quality of life during palliative care and an absence of specific programs in the stage of fast changes that, alone, demand for adaptive efforts.

Descriptores: Adolescent; Neoplasm; Hospice care; Pediatric nursing.

\section{RESUMEN}

Cuidar de adolescentes con cáncer en el proceso de muerte y morirse es un gran desafío para los profesionales de salud, debido al alto costo emocional y las especificidades que involucran esta etapa del desarrollo humano. La finalidad del presente estudio es revisar la producción científica relativa a los cuidados paliativos para adolescentes con cáncer. Se trata de una investigación bibliográfica con dados recolectados en las bases Lilacs, Medline, Psyclnfo y vías no-sistemáticas. A partir del análisis de los textos, fueron identificadas tres temáticas: adolescencia y sus diferentes definiciones; particularidades del adolescente con cáncer y cuidados paliativos para adolescente con cáncer. Se observó que faltan investigaciones basadas en evidencias que definan el panorama de los síntomas Que le afectan la calidad de vida durante los cuidados paliativos y también la ausencia de programas específicos en esa fase de transformaciones rápidas Que, por si solo, demanda esfuerzos adaptativos.

Descriptores: Adolescente; Neoplasia; Cuidados paliativos; Enfermería pediátrica. 


\section{INTRODUÇÃO}

O câncer em crianças e adolescentes, até cerca de duas décadas, era considerado uma doença fatal, mas com os avanços da tecnologia e do cuidado em saúde, atualmente, é uma doença potencialmente curável Quando se tem acesso ao diagnóstico precoce e o tratamento ocorre em centros especializados ${ }^{(1)}$. Estes fatores têm aumentado a sobrevida de crianças e adolescentes com câncer ${ }^{(2,3)}$, porém, mesmo com os progressos verificados nas últimas décadas, aproximadamente $25 \%$ deles não conseguem obter a cura ${ }^{(4)}$.

Quando o tratamento curativo não é mais uma opção, o paciente, a família e a equipe de saúde enfrentam grandes desafios, como o estabelecimento de medidas para o controle da dor e de outros sintomas, acompanhamento e suporte psicossocial e espiritual para a criança, o adolescente e seu entorno familiar, com a finalidade de buscar melhor Qualidade de vida(3).

As Questões relativas às últimas fases da vida e do próprio processo de morte e morrer, atualmente, têm se tornado objeto de reflexão no campo da saúde, da filosofia, da antropologia e sociologia e existe um consenso entre os pesquisadores Quando afirmam Que não se deve preservar a vida biológica a QualQuer custo (obstinação terapêutica) se isso implicar mais dor e sofrimento ao paciente e, sobretudo, perda da auto-estima e dignidade ${ }^{(5)}$. Estas Questões têm sido foco de preocupação e reflexão também da Organização Mundial da Saúde (OMS) Que, em 1990, estabeleceu uma modalidade de assistência denominada cuidados paliativos ${ }^{(6)}$.

A OMS definiu como cuidados paliativos aQueles Que valorizam a vida dos pacientes e familiares ajudando-os a viver a doença na sua fase final, mediante a prevenção e alívio do sofrimento, identificado precocemente. Estabelece, ainda, assistência ampla, com foco no tratamento da dor e de outros problemas físicos, psicossociais e espirituais ${ }^{(6)}$.

O Que leva um paciente a ser incluído num programa de cuidados paliativos é a condição de Que, além do tratamento curativo, existem outros sintomas e desconfortos Que comprometem sua Qualidade de vida; sendo assim, necessitam de uma abordagem competente e especializada. Por sua complexidade, o cuidado paliativo reQuer planejamento interdisciplinar e ação multiprofissional ${ }^{(7)}$.

As enfermeiras têm papel importante nos cuidados paliativos, com particular responsabilidade no provimento de informações, aconselhamento e educação dos pacientes e familiares, principalmente na manutenção do elo domicílio/hospital. Pelo vínculo com os pacientes, são as mais indicadas para monitorar e avaliar a dor e outros sintomas ${ }^{(6)}$.

Diversos autores $^{(4)}$ têm refletido sobre o processo de morte e morrer de adultos e crianças, no entanto, a literatura mostra Que pouco tem sido produzido sobre o processo de morte e morrer de adolescentes e as circunstâncias de suas mortes ${ }^{(1-3)}$. Assim, o cuidar de adolescentes com câncer Que vivenciam a terminalidade da doença tem sido uma das tarefas mais difíceis da medicina, talvez pela escassez de pesquisas sobre o manejo de tais pacientes e devido ao pouco conhecimento Que se tem sobre suas necessidades.

Diante disso, o objetivo do presente estudo é revisar a produção científica nacional e internacional relativa aos cuidados paliativos para adolescentes com câncer, focalizando as particularidades da doença ao longo de seu curso, inclusive o processo de morte e morrer.

\section{METODOLOGIA}

Esta investigação caracteriza-se como pesQuisa bibliográfica ${ }^{(8)}$, pois utilizou como fonte de coleta de dados a bibliografia, entendida como um conjunto de publicações encontrado em periódicos, livrostextos e documentos elaborados por instituições governamentais e sociedades/associações científicas.

A coleta de dados foi realizada nas bases de dados LILACS, MEDLINE, PsycINFO e vias não-sistemáticas, no período de 1990 a 2006. Para tanto, utilizaram-se os descritores "cuidados paliativos e adolescentes" (para as línguas portuguesa e espanhola) e "palliative care and adolescents", "terminal care and adolescents" (para a inglesa). Como critérios de inclusão elegeram-se as publicações em inglês, português e espanhol, na forma de artigos (ensaio, revisão, pesquisa, relato de experiência e estudo de caso), independentemente da formação profissional do autor.

A avaliação inicial do material bibliográfico ocorreu mediante a leitura dos resumos, com a finalidade de selecionar aqueles que atendiam aos objetivos do estudo. Identificou-se um total de 54 publicações, das Quais apenas 16 preenchiam os critérios de inclusão. Dentre as últimas, I não estava disponível no Brasil e outras 4 não foram localizadas em tempo hábil; sendo assim, a amostra final de artigos obtidos de maneira sistemática foi composta por 11 publicações, Que somadas a outras 12 selecionadas de maneira não-sistematizada, totalizaram 23 publicações.

De posse dos artigos, passou-se à etapa seguinte, ou seja, leitura minuciosa, na íntegra, da cada artigo, visando ordenar e sistematizar as informações necessárias para o preenchimento do instrumento de coleta de dados, elaborado para essa finalidade, o Qual continha os seguintes tópicos: fonte de levantamento, dados de identificação, descritores, temática central, abordagem metodológica, instrumentos de coleta de dados e conclusões/considerações finais.

A maioria dos artigos analisados trazia na introdução uma definição cronológica, fisiológica, sociológica ou psicológica sobre a adolescência, e também registrava aspectos e particularidades do adolescente com câncer, mesmo Que o foco fosse apenas os cuidados paliativos. Por estas características, organizou-se o material empírico ao redor de três temáticas: adolescência e suas diferentes definições; particularidades do adolescente com câncer e cuidados paliativos para adolescente com câncer.

\section{RESULTADOS}

Dos 23 artigos analisados, 10 foram localizados na base MEDLINE; I na base LILACS e 12, em lista de referências bibliográficas de artigos seminais. Observou-se variação Quanto ao ano de publicação, destacando-se maior presença do ano de 2004. Os periódicos Que veicularam as publicações analisadas neste estudo tiveram como países de origem Estados Unidos da América (9); Brasil (3); Austrália (3); Finlândia (2); Inglaterra (2); Canadá (2); Escócia (1) e Taiwan (1).

A seguir, encontra-se a apresentação do material empírico, feita a partir das temáticas identificadas:

\section{Adolescência e Suas Diferentes Definições}

Nos textos analisados, a definição de adolescência foi uma constante, como já mencionado; no entanto, tal definição contempla 
as mais diferentes visões. Num estudo de revisão sobre adolescentes com câncer ${ }^{(9)}$, adolescência foi conceituada como um fenômeno culturalmente dependente e de origem recente. Para os autores, em muitas sociedades primitivas, ainda hoje, a adolescência não existe e a transição da infância para a idade adulta ocorre no curso de um dia, geralmente marcada por uma cerimônia ritualística. Segundo os autores, há consenso de que a adolescência é o período entre o início da puberdade e a cessação do crescimento físico, ou seja, dos 11 aos 19 anos de idade.

Um outro estudo ${ }^{(10)}$ apresenta uma diferenciação conceitual entre adolescência e puberdade, indicando Que nesta última ocorre a maturação sexual; já a adolescência compreende o período da maturação sexual até a idade da independência, assegurada por via legal. Ainda acrescenta Que esta etapa é marcada por intensas transformações biopsicossociais.

Na definição da chamada "síndrome da adolescência normal", destaca-se os seguintes componentes: busca de si mesmo e da identidade; tendência grupal; necessidade de intelectualizar e fantasiar; crises religiosas Que podem ir do ateísmo ao misticismo mais fervoroso; deslocalização temporal; evolução sexual manifesta Que vai do auto-erotismo a heterossexualidade; atitude social reinvidicatória com atitudes anti-sociais de diversas intensidades; contradições sucessivas nas condutas; separação progressiva dos pais e constante flutuação de humor e estado de ânimo ${ }^{(1)}$.

A adolescência também foi definida como a fase do estágio de operações formais, ou seja, do desenvolvimento da habilidade de pensar e de usar idéias abstratas ${ }^{(12)}$, etapa de transição complexa da infância para a idade adulta, onde se destaca o desenvolvimento da auto-imagem ${ }^{(2)}$. A percepção da imagem corporal é um aspecto central e intrínseco do desenvolvimento da identidade, assim QualQuer deformidade ou imperfeição, obvia ou não, pode ser inaceitável para o adolescente, causando nele desgosto e vergonha $^{(2,9)}$. Além do desenvolvimento da identidade, existem ainda outras especificidades da adolescência, como: sentir-se confortável com seu corpo; buscar a independência dos pais e de outras figuras de autoridade; construir relações novas e significantes com outros do mesmo sexo e do sexo oposto e desenvolver seu próprio sistema de valores ${ }^{(9)}$.

O Cumulative Index to Nursing e Allied Health Literature descrevem adolescência, para ambos os sexos, como o período compreendido entre 13 e 18 anos de idade; já a Organização Mundial de Saúde considera adolescentes aqueles indivíduos com idade entre 10 e 19 anos, faixa etária Que tem como marco inicial as mudanças corporais da puberdade e como marco final a sua inserção social, profissional e econômica('(12).

$\mathrm{Na}$ análise dos textos, os autores buscaram identificar uma articulação entre a adolescência, apresentada como um período delicado da vida, na Qual o amadurecimento físico e moral e onde as aspirações e planos para o futuro tomam grandes proporções, e como esta etapa é vivida por aqueles Que têm uma doença como o câncer.

\section{Particularidades do Adolescente com Câncer}

Os adolescentes, durante seu desenvolvimento, têm Que lidar com um turbilhão de hormônios Que irão provocar mudanças físicas, emocionais e psicológicas. Esse período de suas vidas torna-se ainda mais difícil Quando se deparam com uma doença como o câncer Que, ainda hoje, causa grande impacto tanto na vida do próprio doente Quanto na de sua família ${ }^{(13)}$. Assim, o câncer na adolescência irá acrescentar novas demandas e novos desafios a esta etapa do ciclo vital ${ }^{(14)}$.

O câncer e seu tratamento, freqüentemente, geram efeitos colaterais, como perda de peso, náusea, vômito, alopecia e fadiga, Que alteram de modo significativo a aparência física do adolescente, prejudicando seriamente o desenvolvimento do senso de identidade e sua imagem corporal ${ }^{(2,9)}$, o Que faz com Que ele se ausente da escola com conseQüente baixo rendimento escolar ${ }^{(12,15)}$. Tais fatores são sentidos e vivenciados não só pelos adolescentes, mas também por suas famílias, Que ainda os têm como crianças ou, no mínimo, como um dependente ${ }^{(16)}$.

Mudanças contínuas ocorrem entre a vida de um adolescente normal, com independência, desenvolvimento da vida social e escolar e a de um adolescente com câncer, Que enfrenta a Quimioterapia e seus efeitos colaterais. Estas mudanças podem causar tensão nestes últimos, pelo fato de a doença os obrigarem a viver em diversos mundos e lugares diferentes, ao mesmo tempo ${ }^{(16)}$.

Estudo $^{(15)}$ com abordagem Qualitativa, realizado na Finlândia, analisou 20 pacientes com câncer com idade entre 13 e 18 anos, utilizando como instrumentos de coleta de dados a entrevista e observação, com a finalidade de identificar como a doença afetou a relação entre os adolescentes e seus pais. Observou-se Que os pais tendiam a ser extremamente cautelosos e protetores, dificultando os processos de independência e de tomada de decisão de seus filhos. Segundo os autores, apesar disso, os pais foram citados como boa fonte de suporte, assim como outros familiares e professores. Os amigos também apareceram como suporte importante, pois, os adolescentes podiam compartilhar com eles suas experiências.

Os autores ${ }^{(15)}$ mencionaram ainda Que os participantes do estudo receberam informações sobre a doença, seu tratamento e efeitos colaterais, assim como sobre as restrições e limitações Que ela poderia causar. Argumentaram, porém, Que na agenda de discussão não constavam informações sobre o Que eles poderiam fazer; como lidar com situações embaraçosas, nem Qual o impacto dos medicamentos e tratamentos nas suas vidas, futuramente. Destacaram, ainda, a importância de atitude positiva dos profissionais de saúde e do respeito à privacidade do adolescente.

Estudo $^{(2)}$ realizado no Canadá, com 5 adolescentes portadores de câncer, com idade entre 14 e 17 anos, teve como objetivo conhecer como eles percebiam as alterações em suas imagens corporais e Qual o impacto que elas causavam em seu cotidiano. A coleta de dados ocorreu mediante entrevistas com análise Qualitativa de dados. $\mathrm{O}$ autor observou Que as alterações físicas modificaram a maneira como os adolescentes se viam, levando-os a se sentirem diferentes de outros adolescentes. Em tais situações, mencionaram Que se sentiram anormais, estranhos e vulneráveis. A importância dos amigos também foi citada por eles, por colaborarem no restabelecimento de suas vidas sociais, fato também mencionado em estudo anterior ${ }^{(15)}$.

Num outro estudo ${ }^{(17)}$ investigou-se o impacto do câncer e dos sintomas a ele relacionados sobre a imagem Que o adolescente faz de si próprio, na trajetória da doença. Identificou-se Que as mudanças Que ocorrem em seus corpos afetam a sua auto- 
percepção, a ponto de mencionarem Que se sentiam incapazes, prisioneiros, inválidos, estranhos e cansados. A autora traz, ainda, as implicações desse conhecimento para a prática de enfermagem, destacando a necessidade de se elaborar um plano de cuidados Que contemple tais aspectos.

Estudo realizado em Taiwan ${ }^{(16)}$, com 16 adolescentes, seus pais e cuidadores, cuja coleta de dados incluiu entrevista e análise Qualitativa dos dados, identificou Que os pais geralmente relutavam em discutir a doença com seus filhos. Apesar disso, esses jovens sabiam Que havia algo errado com eles, e devido à ausência de discussão criavam suas próprias percepções e idéias sobre a doença Que os acometia. Por isso, sentiam medo da rejeição de outras pessoas, ao ficarem sabendo da sua doença. Também referiram Que o Que mais os incomodava era o sofrimento causado por procedimentos invasivos dolorosos, cujas finalidades freqüentemente não lhes eram explicadas, e Que a crescente dor poderia indicar Que sua morte estaria próxima.

Segundo os autores ${ }^{(16)}$, ter amigos com a mesma doença foi mencionado como importante fonte de ajuda para Que os adolescentes se integrassem à experiência do câncer no contexto social e de desenvolvimento de suas vidas. $\mathrm{O}$ fato de poder falar sobre suas próprias experiências com outros Que também a vivenciavam davam-lhes maior segurança.

Estudo $^{(18)}$ realizado com adolescentes em acompanhamento num hospital-escola do interior do estado de São Paulo identificou situações de ambivalência vivenciadas pelo adolescente e seus familiares, seja em relação ao tratamento (repelindo-o e precisando deste), à família (contestando a superproteção e solicitando acolhimento), aos amigos (afastando-se deles e lamentando seu afastamento), e, mesmo à morte; falam sobre esta possibilidade, mas não conseguem imaginá-la como sua. Num momento em Que os adolescentes com câncer já enfrentam as dificuldades de lidar com suas perdas físicas (seu corpo infantil) e psíquicas (perda da identidade dos pais) se deparam, ainda, com a ameaça concreta de sua finitude.

\section{Cuidados Paliativos para Adolescente com Câncer}

Lidar com um prognóstico terminal pode interromper as atividades cotidianas e as fases de desenvolvimento dos adolescentes, no entanto, é importante Que eles tenham a oportunidade de continuar sua educação, mesmo na fase terminal da doença ${ }^{(19)}$.

Os jovens, nestas condições, podem desejar expressar sentimentos e medos sobre a morte ou o Que está por trás dela ${ }^{(16)}$. Alguns manifestam medo de serem esquecidos depois da morte; neste caso, encorajá-los a escrever uma lista de atividades a serem desenvolvidas a curto prazo, planejar seu próprio funeral e gravar uma fita de áudio ou vídeo para deixar para seus parentes e amigos pode ajudá-los a lidar com a situação ${ }^{(19)}$.

Uma vez que a decisão sobre o tratamento tenha sido tomada, devem se escolher os elementos dos cuidados paliativos a serem implementados, devendo a transição entre o tratamento curativo e o paliativo ocorrer de modo gradual. Estas escolhas incluem o controle de sintomas (medicações e outras intervenções específicas), o local do cuidado e da morte (casa, hospital ou outro lugar) e os responsáveis pelos cuidados paliativos (equipe hospitalar, família, e/ou outros) ${ }^{(20)}$.
As freqüentes hospitalizações reforçam o isolamento social vivenciado por muitos pacientes terminais ${ }^{(19)}$, Que se traduzem em isolamento de seus pais, familiares e comunidade, de outros jovens em condições semelhantes e da própria equipe de saúde ${ }^{(15)}$.

Quando um adolescente está morrendo, seus pais podem ter exacerbado sentimentos de perda e culpa, pois as aspirações de imortalidade de seu filho estão se desintegrando e, muitas vezes, eles se sentem responsáveis pela situação vivida ${ }^{(21)}$ e incapacitados de proteger o filho ${ }^{(3)}$.

Depois da morte de um adolescente, as famílias freqüentemente reportam que seu luto não é só pelo sentimento da perda do filho, mas também por sentimentos de isolamento da equipe de saúde e da familiaridade do hospital ${ }^{(4)}$. Durante o luto, Que é uma resposta normal à perda, nem todas as pessoas precisam de ajuda para lidar com esse sentimento, porém, Quando necessário, essa intervenção deve se estender desde o cuidado terminal até depois da morte ${ }^{(22)}$.

Pesquisa ${ }^{(23)}$ realizada com 103 famílias americanas cujas crianças tinham morrido com câncer encontrou Que $89 \%$ dos pais perceberam Que seus filhos vivenciaram intensa dor $(>80 \%)$ tanto física, emocional e espiritual, além de outros sintomas angustiantes, como fadiga (>95\%) e dispnéia (>80\%), um mês antes da morte. Durante as últimas 48 horas, pacientes vivenciam aumento da fracueza e imobilidade, perda do interesse pela comida e bebida, dificuldade para deglutir e adormecer. A prioridade, então, é o controle dos sintomas e suporte familiar ${ }^{(24)}$.

O controle da dor é um fator crucial nos cuidados paliativos ${ }^{(19)}$, pois ela pode ser incapacitante e causar efeitos indesejados no jovem e na família. Seu manejo pode ser feito através de intervenções farmacológicas e não-farmacológicas; dentre as primeiras, destaca-se a administração de opióides, Que, apesar do efeito colateral como depressão respiratória, não deve ter seu uso limitado no esforço de aliviar a dor ${ }^{(25)}$.

Estudo realizado nos Estados Unidos da América, com 39 pais de crianças Que morreram com câncer, e também com 16 médicos, 3 enfermeiras e 2 capelães, evidenciou Que os pais classificaram de difíceis algumas decisões a serem tomadas, como: manter ou tirar suporte de vida, fazer Quimioterapia paliativa ou não e escolher entre uma ordem de ressuscitar ou não. Identificaram, ainda, alguns fatores Que influenciaram na tomada de decisão: tipos de informação recebida dos profissionais de saúde, Que incluíam fatos, explanações e opiniões sobre a doença, habilidades funcionais, probabilidade de sobrevivência e as complexidades de um cuidado continuado. Para os profissionais de saúde, influenciaram mais freeüentemente na tomada de suas decisões: o respeito às preferências do paciente e da família e a conclusão de Que o paciente não sobreviveria ${ }^{(1)}$.

Pesquisa realizada também nos Estados Unidos da América com 52 pais; I adolescente de 18 anos de idade, responsável pelas suas próprias decisões; 9 adolescentes com idade entre 15 e 17 anos e 22 médicos, identificou, a partir de entrevistas, Que os adolescentes consideraram na decisão do fim da vida, os seguintes aspectos: recomendações, preferências ou opiniões dos profissionais da saúde e membros da família $(n=14)$; relatos da sobrevida de pessoas Que ficaram em ventilação mecânica ou Que utilizaram outra técnica para prolongar a vida $(n=13)$; crenças religiosas e na vida após a morte $(\mathrm{n}=8)^{(1)}$.

A avaliação da Qualidade do cuidado prestado aos filhos com câncer, no fim da vida, foi objetivo de um estudo ${ }^{(26)}$ Que teve como 
população pais e médicos responsáveis por esse cuidado. Para os pais, a comunicação médico-paciente sensível e cuidadosa foi a principal determinante da alta Qualidade do cuidado; já para os médicos, a maior Qualidade do cuidado baseou-se no melhor controle da dor e no menor tempo de estadia do adolescente no hospital; nenhum deles mencionou fatores relativos à comunicação, como importantes. Portanto, há Que se olhar para as diferentes perspectivas dos sujeitos envolvidos ao se elaborar o planejamento do cuidado.

As circunstâncias especiais em Que se encontram os adolescentes terminais devem ser enfatizadas de diferentes modos: primeiro, para esses pacientes não há perspectiva para autonomia a longo prazo, como para os adolescentes saudáveis, esperando recuperarem-se de uma condição aguda; a única forma de autonomia para eles é aQuela exercida no seu cotidiano, no dia presente. Segundo, parece difícil afirmar algo que vá aumentar os direitos de menores em circunstâncias extraordinárias de doença terminal, uma vez Que as noções de bem-estar e autonomia podem tomar diferentes significados; terceiro, no respeito à autonomia de um menor, funcionalmente competente, não é incoerente o envolvimento da família e não necessariamente implicam intervenções de terceiros ${ }^{(20)}$.

\section{DISCUSSÃO}

Poucas são as pesquisas sobre cuidados paliativos direcionados aos adolescentes com câncer, e as existentes, geralmente, foram realizadas nos Estados Unidos da América ou em países da Europa.

Há consenso de Que o câncer em um adolescente modifica radicalmente sua vida e a de sua família. A confirmação do diagnóstico, a terapêutica e a notícia do sucesso ou não do tratamento fazem surgir nas pessoas envolvidas uma expectativa particular em cada uma destas etapas. A tarefa de acompanhar um adolescente durante a trajetória da doença exige compreensão das diferentes respostas emocionais; em outras palavras, confirmado o diagnóstico, a interpretação feita pelo doente e sua família é a da sentença de morte Que, ao mesmo tempo, impõe a busca pela cura. Com o sucesso do tratamento, inicia-se o período de apreensão; assim, a cada consulta de revisão ou controle vem o medo de receberem notícias desalentadoras. Em caso de recidiva, o ciclo será reiniciado desde Que haja tratamento a ser instituído; do contrário, virá a etapa da terminalidade da doença ${ }^{(27)}$.

Com a doença em evolução, o adolescente e sua família deverão cumprir duas tarefas: a primeira de foro intrapsíquico, Quando se despedem de si mesmos; a segunda, Quando suas vidas e relações interpessoais são checadas de várias formas. Relações sociais, espirituais e resoluções de projetos de vida, na medida do possível, devem ser finalizadas ${ }^{(27)}$. Particularmente nesta etapa acompanhar o adolescente é extremamente estressante, tanto sob o ponto de vista emocional Quanto prático.

Adolescentes em acompanhamento nos cuidados paliativos devem ter sob controle aspectos, como administração de analgésicos e sedativos, anti-eméticos e outras medicações para alívio de seus sintomas; Quanto aos Que desejarem ficar nas suas casas, devem ter esta permissão. Ainda, devem ser incluídos nos processos de tomada de decisão. A equipe de saúde deve assegurar Que a efetividade da filosofia do cuidado paliativo não seja seriamente comprometida por tais escolhas.

Lidar com a morte não é fácil e é muito doloroso Quando se constatam um futuro perdido e a injustiça da doença, especialmente de um adolescente. No entanto, a maneira de tornar esse processo menos estressante para todos os envolvidos é enfrentar a realidade e propor medidas de alívio da dor e do sofrimento, tanto para o adolescente Quanto para família e equipe de saúde.

Dá-se um sentido positivo à condição terminal Quando a criança ou adolescente se sente Querido; especialmente nessa situação, a equipe médica e de enfermagem, os amigos e familiares não devem se distanciar deles, mas, sim, acompanhá-los durante todas as fases do processo diagnóstico e terapêutico, inclusive na morte e no luto.

Diante da complexidade do processo de cuidar do adolescente com câncer, particularmente na fase dos cuidados paliativos, a equipe de saúde ao praticar a compaixão e a solidariedade e ao the oferecer apoio contribui para minimizar o impacto da doença e aliviar seu sofrimento, ajudando-o, assim, a construir novas perspectivas para enfrentar os problemas cotidianos.

Nesta perspectiva, é fundamental Que o planejamento do cuidado ao adolescente com câncer seja estabelecido pelas equipes de saúde, juntamente com o próprio adolescente e seus familiares, contemplando os aspectos físicos e emocionais, seus valores culturais e éticos, tradição religiosa, transcendendo, portanto, condutas dirigidas somente aos sinais e sintomas físicos. A equipe de saúde deve, então, considerar como relevantes para essa faixa etária, a necessidade de preservar a privacidade, a relação com os pares e o contato com o ambiente externo.

Este é um desafio para as equipes de saúde responsáveis pelos cuidados paliativos, pois elas devem promover mudanças no sentido de abandonar sua visão paternalista de Que é a detentora do saber e única responsável pela tomada de decisões. Elas devem, então, inserir o adolescente e família no processo, adotando-se uma abordagem mais participativa e simétrica, possibilitando Que estes possam propor intervenções Que melhorem aspectos da Qualidade de suas vidas.

Algumas barreiras têm sido identificadas no sentido de impedir a implementação adeQuada de cuidados paliativos para crianças e adolescentes com câncer. Dentre elas, estão a relativa raridade desta morbi-mortalidade; os diferentes estágios do desenvolvimento dos 0 aos 19 anos de idade, Que requerem intervenções diferenciadas; a falta de pesquisas baseadas em evidências que definam o panorama dos sintomas Que afetam a Qualidade de vida do adolescente, durante o tratamento e no estágio final, e Quais suas causas; a falta de programas educativos voltados para formação dos profissionais na área da saúde, visando implantar medidas efetivas para melhorar a Qualidade de vida dos pacientes e suas famílias, e ainda falta de investimentos adequados para se dispensarem os cuidados paliativos no domićlio dos clientes. Para transpor estas barreiras, é necessário que os profissionais envolvidos nos cuidados paliativos possam se dedicar à pesquisa e defender essa área com a mesma dedicação Que se dispensam aos estudos para terapias curativas ${ }^{(28)}$.

\section{CONSIDERAÇÕES FINAIS}

Programas de cuidados paliativos têm sido reconhecidos como estratégias fundamentais para melhorar a Qualidade de vida de 
pessoas, cujas doenças as levam a vivenciar a fase terminal. Controle dos sofrimentos físico, emocional, espiritual e social é essencial nesta modalidade de cuidado, Que tem a pessoa doente (desde o diagnóstico até o momento da morte) e sua família (durante o curso da doença e em programas de enlutamento) como focos de atenção na busca pela melhor Qualidade de vida.

Cuidados paliativos encontram-se em um campo conceitual, metodológico e instrumental ainda em construção, sendo assim, sua definição, bem como suas estratégias a partir da prática são um desafio para as equipes. Nos cuidados paliativos a ação não é movida apenas pela competência técnico-científica, apoiada no processo diagnóstico e terapêutico; a ação é, também, determinada por Questões políticas, éticas, culturais, sociais e subjetivas, assim, o desafio das equipes é encontrar no trabalho cotidiano, junto aos Que recebem cuidados paliativos, um equilíbrio harmonioso entre a razão e a emoção.

Ressaltamos a urgência de se intensificar as investigações sobre cuidados paliativos para adolescentes com câncer, com objetivo de trazer subsídios Que permitam viabilizar a introdução dessa prática nos serviços de saúde, e ainda, Que os gestores e produtores de políticas públicas utilizem tais evidências científicas no planejamento das ações em saúde.

\section{REFERÊNCIAS}

I. Hinds PS, Oakes L, Furman W, Quargnenti A, Olson MS, Foppiano $\mathrm{P}$, et al. End-of-life decision making by adolescents, parents, and healthcare providers in pediatric oncology. Cancer Nurs 2001; 24(2): 122-34.

2. Larouche SS, Chin-Peuckert L. Changes in body image experienced by adolescents with cancer. I Pediatr Oncol Nurs 2006; 23(4): 200-9.

3. Hayout I, Krulik T. A test of parenthood: dilemma of parents of terminally ill adolescents. Cancer Nurs 1999; 22(1): 7I-9.

4. Hurwitz CA; Duncan J; Wolfe J. Caring for the child with cancer at the close of life. JAMA 2004; 292(17): 2141-9.

5. Pessini L. A filosofia dos cuidados paliativos: uma resposta diante da obstinação terapêutica. Mundo da Saúde 2003; 27(27): 15-32.

6. World Health Organization. Cancer pain relief and palliative care. Geneva: WHO; 1990.

7. World Health Organization. National cancer control programs: polices and management guidelines. $2^{\text {nd }}$ ed. Geneva: WHO; 2002.

8. Moreira H, Caleffe LG. Metodologia da pesquisa para o professor pesquisador. Rio de Janeiro: DP\&A; 2006.

9. Whyte F, Smith L. A literature review of adolescents an cancer. Eur I Cancer Care 1997; 6: 137-46.

10. Ribeiro IB; Rodrigues BMRD. Cuidando de adolescentes com câncer: contribuições para o cuidar em enfermagem. Rev de Enfermagem UERJ 2005; 13: 340-6.

11. Knobel M. Adolescência normal. $7^{\text {a }}$ ed. Porto Alegre: Artes Médicas; 1988.

12. Thomas DM. Adolescents an young adult cancer: a revolution in evolution? Int Med I 2006; 36: 302-7.

13. Klopfenstein K. Adolescents, cancer, and hospice. Adolescent Medicine 1999; 10(3):437-43.

14. Menossi MJ, Lima RAG. A problemática do sofrimento: percepção do adolescente com câncer. Rev Esc Enferm USP 2000; 34(1): 45-51.
15. Hokkanen H, Eriksson E, Ahonen O, Santera S. Adolescents with cancer: experience of life and how it could me made easier. Cancer Nurs 2004; 27(4): 325-35.

16. Yeh $\mathrm{CH}$. Life experience of taiwanese adolescents with cancer. Scan I Caring Sci 2002; 1 6: 232-9.

17. Woodgate RL. A different way of being: adolescents' experiences with cancer. Cancer Nurs 2005; 28(1): 8-15.

18. Bessa LCL. "Adolescer" do paciente com câncer [dissertação]. Ribeirão Preto: Faculdade de Filosofia, Ciências e Letras de Ribeirão Preto da Universidade de São Paulo; 1997.

19. Carr-Gregg MRC, Sawyer SM, Clarke CF, Bowes G. Caring for the terminally ill adolescents. Med I Austr 1997; 166: 255-8.

20. Nitschke R, Meyer WH, Huszti HC. When the tumor is not the target, tell the children. I Clin Oncol 2001; 19: 595-6.

21 . Klopfenstein $\mathrm{KJ}$. Variables influencing end-of-life care in children and adolescents with cancer. J Pediatric Hematol/Oncol 200 I; 23(8): 48I-6.

22. Maddocks I. Grief and bereavement. Med I Austr 2003; 179 : S6-S7.

23. Wolfe J, Grier HE, Klar N. Symptoms and suffering at the end of life in children with cancer. N Engl I Med 2003; 342: 32633.

24. Adam I. ABC of palliative care: the last 48 hours. BMJ 1997; 315: 1600-3.

25. Hooke C, Hellsten MB, Stutzer C, Forte K. Pain management for the child with cancer in end-of-life care: APON position paper. Pediatr Oncol Nurs 2002; 19(2): 43-7.

26. Mack JW, Hilden JM, Watterson I, Moore C, Turner HEG, Weeks IC, Wolfe I. Parent and physician perspectives on Quality of care at the end of life in children with cancer. I Clin Oncol 2005; 23(36): 9155-61.

27. Kovács MJ. Autonomia e o direito de morrer com dignidade. Bioética 1998; 6.

28. Harrys MB. Palliative care in children with cancer: which child and when? I Nat Cancer Inst Monogr 2004; 32: 144-9. 